\title{
Competencia emocional del futuro docente en educación infantil y primaria: análisis de predictores significativos
}

\author{
María P. Sepúlveda-Ruiz¹, Francisco D. Guillén-Gámez², Elena García-Vila¹ y María J. Mayorga-Fernández ${ }^{1 *}$ \\ (1) Universidad de Málaga, Facultad de Ciencias de la Educación, Departamento de Didáctica y Organización Escolar, \\ Málaga-España (correo-e: mdsepulveda@uma.es; elenavila@uma.es; mjmayorga@uma.es) \\ (2) Universidad de Córdoba, Facultad de Ciencias de la Educación, Departamento de Didáctica y Organización Escolar, \\ Córdoba-España (correo-e: dguillen@uco.es)
}

${ }^{*}$ Autor a quien debe ser dirigida la correspondencia.

Recibido Nov. 20, 2020; Aceptado Ene. 19, 2021; Versión final Feb. 15, 2021, Publicado Jun. 2021

\begin{abstract}
Resumen
El objetivo del presente trabajo es analizar y comparar descriptiva y estadísticamente el nivel de competencia emocional de los/as futuros maestros/as de los grados en educación infantil y educación primaria, así como conocer la incidencia de diversas variables académicas y personales en el desarrollo de dicha competencia emocional. Para ello, se plantea un estudio con una metodología ex post facto descriptiva e inferencial con una técnica de encuesta. Los resultados muestran que los/as estudiantes poseen un nivel de desarrollo emocional medio, destacando la influencia positiva en el nivel de desarrollo emocional de estos en variables como: las calificaciones, el esfuerzo hacia el estudio y la motivación. Teniendo en cuenta los resultados obtenidos, se puede concluir que es fundamental que las instituciones de educación superior introduzcan la formación emocional en los planes de estudio oficiales.
\end{abstract}

Palabras clave: competencias emocionales; formación inicial docente; desarrollo profesional; rendimiento académico

\section{Emotional competence of prospective early childhood and primary education teachers: analyses of significant predictors}

\begin{abstract}
The aim of this study is to analyze and compare descriptively and statistically the level of emotional competence of prospective teachers enrolled in the degrees of early childhood education and primary education. This study also intends to assess the incidence of academic and personal variables in the development of prospective teachers' emotional competence. An ex-post-facto descriptive and inferential methodology is proposed. The results show that prospective teachers have an average level of emotional development. Variables such as academic performance, effort towards studying, and motivation are prominent because of their positive influence on emotional development. Based on the results obtained, it can be concluded that it is essential for higher education institutions to introduce emotional training in their academic degree programs.
\end{abstract}

Keywords: emotional competencies; initial teacher training; professional development; academic performance 


\section{INTRODUCCIÓN}

La formación inicial y el desarrollo profesional docente en la sociedad actual, y en los contextos de trabajo donde se desenvuelve su actividad profesional, requiere en estos momentos que se preste especial atención a las competencias emocionales del maestro/a, entendidas como "el conjunto de conocimientos, capacidades, habilidades y actitudes necesarias para comprender, expresar y regular de forma apropiada los fenómenos emocionales" (Bisquerra \& Pérez, 2007, p.69). Estas ocupan un lugar prioritario para adaptarse de modo inmediato a las necesidades y demandas educativas que se planteen, responder de modo adecuado a las diferentes situaciones socio-laborales y saber estar, entendido éste como un dominio del entorno a nivel interpersonal e intrapersonal (Ávila, 2019), y afrontar la profesión docente con herramientas suficientes para ofrecer una educación de calidad. Se puede afirmar que las competencias emocionales están constituidas por 5 dimensiones: conciencia emocional, regulación emocional, autonomía personal, inteligencia interpersonal, habilidades de vida y bienestar (Bisquerra \& Pérez, 2007), por lo que se configuran en base a un modelo basado en el constructo inteligencia emocional junto a atributos emocionales y sociales (Bisquerra, et al., 2015). Por ello, es prioritario incluir el ámbito emocional en la formación inicial de los/as docentes (Palomera et al., 2019; Pérez-Escoda et al., 2019; Schonert-Reichl, 2019), para que, desde los primeros años de formación, dichos docentes, puedan identificar, comprender y regular sus propias emociones y las de su alumnado, facilitando el aprendizaje de estrategias para reflexionar sobre el desarrollo de la práctica docente.

Por tanto, la regulación emocional es clave en dicha práctica docente, y es por ello, que debe prestarse especial atención en la formación inicial para prevenir posibles situaciones de estrés, ansiedad y relativizar problemas académicos (Del Rosal et al., 2018). También es importante destacar que los profesores/as emocionalmente competentes pueden crear entornos más afectivos y seguros de aprendizaje, promover la motivación intrínseca de los estudiantes, la cooperación, estrategias pacíficas de resolución de conflictos, comunicación positiva, entre otros aspectos, todo lo cual repercute directamente en el aprendizaje del alumnado (Humphries et al., 2018) y en su desarrollo emocional (Denham \& Bassett, 2019), porque el "buen docente es aquel que sabe captar las emociones de sus alumnos y es capaz de conducirlas hacia el éxito en sus vidas" (Palomares-Ruíz \& Serrano-Marugán, 2016, p.25).

En este contexto, existen estudios que afirman que aquellos profesionales cuyas necesidades psicológicas básicas están satisfechas y tienen bien desarrolladas sus competencias emocionales, su estilo docente está más centrado en promover una enseñanza motivadora, priorizar la autonomía de sus estudiantes y conseguir mayor afinidad hacia los mismos, es decir, poseen estilos docentes asertivos (Ávila, 2019; Moè \& Idit, 2020). Por ello, y coincidiendo con los resultados obtenidos por Rosa et al. (2015), Estrada et al. (2016), Maguire et al. (2017) y Aspelin (2019) resulta prioritario introducir programas específicos de Educación Emocional en la formación universitaria, en general, y más concretamente en la formación inicial de los futuros docentes de Educación Infantil y Educación Primaria, puesto que va a permitir a dicho profesorado hacer frente a los retos que socialmente se plantean en la actualidad: capacidad para tolerar la frustración, postergar la recompensa e inmediatez, poseer una actitud crítica para decidir según las circunstancias y el contexto, y sobre todo desarrollar competencias de adaptación, superación y realización intra e interpersonal (Rueda \& Filella, 2016).

En este sentido, para trabajar, conocer y fortalecer esta capacitación en competencias emocionales en la formación inicial es necesario en primer lugar, conocer cómo influye el dominio afectivo en los futuros docentes de Educación Infantil y Primaria, es decir, saber e identificar las competencias emocionales de dichos docentes para poder mejorarlas (Del Rosal et al., 2018) constituyendo esto una de las principales finalidades del presente estudio. Para ello, se han realizado numerosas investigaciones en las que se indaga y trata de comprender el nivel de inteligencia emocional de estos estudiantes.

En la literatura escrita en los últimos años se aprecia diferentes estudios que afirman que el nivel de competencia emocional del alumnado universitarios es medio-bajo (Barraza \& González, 2016). Por ejemplo, Catacora (2020), confirma que las competencias emocionales de los estudiantes de educación superior se encuentran en un nivel bajo y con tendencia a la baja (25.2\% y $27 \%$ ). Si se centra el interés en las titulaciones relacionadas con la Facultad de Educación, Hernáez \& Fernández (2019) realizaron un estudio en el que participaron 366 estudiantes de los Grados de Maestro/a en Educación Infantil y Primaria, concluyendo que los futuros maestros/as de Primaria poseían niveles favorables en competencias emocionales. Sin embargo, esos niveles eran significativamente más bajos en comparación con los futuros profesores/as de Educación Infantil.

Al considerarse las competencias emocionales como elementos fundamentales para la adaptación de los individuos a la sociedad, se han realizado diferentes investigaciones que analizan las relaciones entre competencias emocionales y/o inteligencia emocional y diferentes variables académicas y demográficas. Respecto al rendimiento académico, Del Rosal et al. (2018) comprobó que existían correlaciones significativas en el nivel de inteligencia emocional y el rendimiento académico de los futuros maestros y maestras de Educación Infantil y Primaria, hallando una relación positiva entre ambas variables, es decir, a mayor 
Inteligencia Emocional mayores calificaciones, obteniendo los estudiantes del Grado en Educación Primaria mejores efectos. En cambio, Deighton et al. (2019) obtuvieron resultados contrarios a los anteriores, puesto que en un estudio realizado con 60 estudiantes de primer curso universitario los resultados no mostraron efectos significativos de la inteligencia emocional sobre las calificaciones ni el rendimiento de los estudiantes.

Respecto a la variable género y edad, Dumitriu et al. (2014) analizaron las competencias emocionales de 210 estudiantes universitarios, concluyendo que dicho nivel varía significativamente en ambas variables. Para estos autores, la maduración biológica, intelectual y social del individuo hace que aumente su desarrollo emocional, teniendo una mejor percepción de sí mismos, autocontrol y empatía. Anastasiou (2020) por su parte, destaca en su estudio que los docentes de Educación Infantil poseen un nivel de desarrollo emocional medio, no existiendo efecto de la edad en dicho desarrollo emocional. Resultados similares que corroboran Andrade et al. (2016) y Gutiérrez (2020).

En el mismo contexto, Pegalajar \& López (2015) con estudiantes del Grado en Educación Infantil pusieron de manifiesto que poseían, en general, niveles favorables de autorrealización y desarrollo personal, destacando los resultados en aspectos relacionados con la sociabilidad, comunicación, respeto, colaboración y ayuda. Además, se observaron diferencias significativas respecto al género, siendo más favorables en el alumnado femenino. Del Rosal et al. (2016) comprobaron que el nivel de inteligencia emocional (atención, claridad y reparación emocional) del alumnado matriculado en el Grado en Educación Primaria era mejor que los que realizaban otros Grados centrados en el ámbito de las Ciencias. Concretamente, mostraron diferencias significativas en la dimensión reparación emocional en función del género y grado. Los hombres obtuvieron mejores resultados que las mujeres en esta dimensión, y concretamente los estudiantes que realizaban el Grado de Maestro en Educación Primaria destacaban tanto en lo que respecta a la atención como a la claridad emocional. Del Rosal et al. (2018) en su trabajo con estudiantes de los Grados en Educación Primaria y en Educación Infantil también obtuvieron diferencias significativas entre la variable género y el nivel de inteligencia emocional, y concretamente en las dimensiones "claridad y reparación emocional", siendo superior en el alumnado masculino. Sin embargo, en la dimensión atención emocional eran las alumnas las que destacaban.

Por otro lado, también existen diferentes estudios que se han centrado en analizar los factores incidentes en el desarrollo de las emociones. En este sentido, Trigueros et al. (2019) concluyeron que las emociones positivas influyen en la automotivación, y esta a su vez en el rendimiento académico, por tanto, el desarrollo emocional puede ser un predictor del rendimiento, puesto que las emociones aumentan la tendencia a obtener buenas calificaciones, conclusiones que se corroboran con las obtenidas por Bimayu et al. (2020) y Zhoc et al. (2020). En la misma línea, y obteniendo resultados muy similares, Chinyere \& Afeez (2019) afirman que la inteligencia emocional se asocia significativa y positivamente con el nivel de motivación académica y la actitud hacia los estudios, además de estar estrechamente relacionada con la autorrealización y autoeficacia (Ordun \& Akün, 2017).

Teniendo en cuenta los estudios destacados anteriormente que aproximan al conocimiento de las variables que pueden estar incidiendo en el nivel de competencias emocionales del alumnado de los Grados en Maestro en Educación Infantil y Primaria, y la importancia de vivir una formación significativa y de bienestar, los objetivos que se plantean con el presente estudio son los siguientes: (1) analizar y comparar el nivel de competencia emocional de los futuros maestros/as de Educación Infantil y de Educación Primaria; y (2) conocer la correlación entre diversas variables académicas y personales con el desarrollo de dicha competencia emocional.

\section{MÉTODO}

Esta investigación se engloba en lo que se denominan diseños no experimentales de tipo descriptivo y correlacional con metodologías ex post facto, a través de un cuestionario de autoinforme, realizado a principios del segundo semestre del curso 2019-2020. El propósito de este diseño no es modificar ninguna variable objeto de estudio, sino explorar su naturaleza en la muestra de estudiantes seleccionados, así como estudiar las relaciones que se expresan entre ellas.

\section{Participantes}

Para la recolección de los datos, se ha utilizado un muestro no probabilístico de manera intencional, con un total de 359 estudiantes de la Facultad de Ciencias de la Educación de la Universidad de Málaga y de la Universidad de Almería, ambas procedentes de España. Específicamente, el $63.2 \%(n=227)$ estudiaban primer curso del Grado de Maestro en Educación Infantil con una media de edad de 19.59 años, mientras que el $36.8 \%(n=132)$ estudiaban primer curso del Grado de Maestro en Educación Primaria con una media de 19.22 años. 


\section{Instrumento}

Para medir la competencia emocional se emplearon 21 ítems de los 48 ítems procedentes del Cuestionario de Desarrollo Emocional de Adultos (QDE-A), el cual fue elaborado por Escoda et al. (2010). La elección de estos ítems fue debido a los resultados encontrados al analizar las propiedades psicométricas del instrumento al realizar los correspondientes análisis factorial exploratorio (AFE) y análisis factorial confirmatorio (AFC). Los ítems seleccionados cumplieron los criterios marcados por estos análisis factoriales.

El instrumento estaba organizado en cinco dimensiones: conciencia emocional (capacidad para tomar conciencia de las propias emociones y las de los demás, incluyendo la habilidad para captar el clima relacional en un contexto determinado), regulación emocional (capacidad para utilizar las emociones de forma adecuada. Implica tomar conciencia de la relación entre emoción, cognición y comportamiento), autonomía emocional (capacidad de desarrollo de la autoestima, actitud positiva ante la vida, responsabilidad, capacidad para analizar críticamente las normas sociales, capacidad para buscar ayuda y recursos, autoeficacia personal), competencias sociales (capacidad para mantener buenas relaciones con otras personas), competencias para la vida y el bienestar (capacidad para adoptar comportamientos adecuados y responsables para la solución de problemas personales, familiares, personales y sociales, orientados hacia la mejora del bienestar de la vida personal y social).

Para la medición de la competencia emocional, fue empleada una escala Likert de 5 puntos, donde el valor 1 indicaba "totalmente en desacuerdo", mientras que el valor 5 "totalmente de acuerdo", teniendo en cuenta que cada valor de la escala hace referencia a los siguientes niveles progresivos: valor 1 (competencia muy baja), valor 2 (competencia baja), valor 3 (competencia media), valor 4 (competencia alta) y valor 5 (competencia muy alta). Además, se tuvieron en consideración otras variables como edad, calificación de acceso al grado, calificación media del primer semestre (valoradas mediante un número), esfuerzo y motivación (valoradas en una escala Likert de 10 puntos, donde 1 es nada satisfecho y 10 altamente satisfecho).

El instrumento original fue administrado a una muestra de 1.537 personas con edades comprendidas entre los 17 y los 64 años de edad. Éste presentaba propiedades psicométricas en relación a la fiabilidad y validez de constructo a través del AFE. No obstante, no presentaba resultados sobre el AFC ni tampoco estaba focalizado únicamente en estudiantes de educación. Por ello, dichos valores fueron medidos de nuevo para justificar la fiabilidad y validez del citado instrumento.

\section{Procedimiento y técnicas de análisis}

El análisis de los datos consistió en tres procedimientos: el primero, la verificación de las propiedades psicométricas del instrumento a través de la fiabilidad (alfa de Cronbach), AFE y AFC. En segundo lugar, un análisis descriptivo a través de valores de tendencia central y de dispersión (media y desviación típica) de las dimensiones e ítems del instrumento. En tercer lugar, un análisis comparativo para conocer si existían diferencias significativas en la competencia emocional entre ambas distribuciones (educación infantil y educación primaria). Y, por último, un análisis correlacional para identificar aquellas variables significativas que tienen un efecto en el desarrollo de dicha competencia.

\section{RESULTADOS}

En este apartado se analizan, en primer lugar, las propiedades psicométricas del instrumento de recogida de información, para posteriormente realizar un análisis descriptivo y estadístico de la competencia emocional del alumnado y, por último, se muestran los resultados obtenidos entre las correlaciones realizadas.

\section{Propiedades psicométricas del instrumento}

El AFE fue llevado a cabo con el método de máxima verosimilitud con rotación oblimin. Los valores de la prueba KMO (Kaiser-Meyer-Olkin) fue 0.804 y el test de Bartlet fue significativo $\left(X^{2}=1772.184, p .<0.05\right)$. Fueron eliminados todos aquellos ítems que obtuvieron valores inferiores a 0.3 o que saturaron en otras dimensiones, encontrando un instrumento final de 21 ítems clasificados en cinco factores. La versión final del instrumento explicó el $62.08 \%$ de la puntuación verdadera de los sujetos. En la tabla 1 puede observarse la proporción de varianza que explica cada factor. Por otro lado, el AFC apoyó la estructura factorial formulada en el AFE, formada por cinco variables latentes correlacionadas. El modelo de ecuación estructural fue realizado con el software AMOS V.24 y SPSS V.24. Para la realización del AFC, se calcularon los siguientes valores teniendo en consideración los criterios de Bentler (1989): razón de chi-cuadrado sobre los grados de libertad (X2/g.l; CMIN / DF) teniendo en cuenta que los valores inferiores a 3 indican un buen ajuste; el índice de ajuste comparativo (CFI), el índice de Tucker-Lewis y el índice de ajuste incremental (IFI) considerando valores superiores a 0.90 como un buen ajuste; el Raíz cuadrada media residual (RMR) considerando valores inferior o igual a 0.05 como muy satisfactorio; y por último, el error cuadrático medio de aproximación (RMSEA, 
.058) donde los valores por debajo de 0.06 indican un buen ajuste del modelo. En la tabla 1 se muestran las propiedades psicométricas específicas del AFC, encontrándose unos coeficientes aceptables. Además, se examinó la fiabilidad del instrumento a través de alfa de Cronbach, encontrando un valor satisfactorio $(\alpha=$ $0.73)$.

Tabla 1: Resultados factoriales exploratorios y confirmatorios

\begin{tabular}{|c|c|c|c|c|c|c|c|}
\hline Coeficientes del modelo AFC & $X^{2}$ & $p$. & $C F I$ & $T L I$ & $I F I$ & $R M R$ & $R M S E A$ \\
\cline { 2 - 8 } & 1.669 & 0.001 & 0.954 & 0.940 & 0.955 & 0.050 & 0.043 \\
\hline Coeficientes del modelo AFE & $\begin{array}{c}\text { Varianza verdadera } \\
\text { explicada (\%) }\end{array}$ & Dim. 1 & Dim. 2 & Dim. 3 & Dim. 4 & Dim. 5 \\
\cline { 3 - 8 } & 23.930 & 14.690 & 8.606 & 8.180 & 6.677 \\
\hline
\end{tabular}

\section{Análisis en la competencia emocional del alumnado}

En la tabla 2 se muestran los valores descriptivos de los ítems seleccionados. En la dimensión Competencia de vida y bienestar todos los ítems tienen una valoración media-alta, pudiendo destacarse la puntuación obtenida por el ítem "Me siento una persona feliz" que en el Grado en Educación Primaria posee una valoración alta, aunque ligeramente superior a los estudiantes del Grado en Educación Infantil. Respecto a la dimensión autonomía emocional, se puede apreciar con claridad que tanto en el Grado en Educación Infantil como en el Grado en Educación Primaria todos los ítems poseen una valoración media-baja, aunque es de destacar que los ítems que la conforman están formulados en negativo. A pesar de ello el ítem con mayor puntuación tanto en uno como en el otro Grado es el relativo a "Me siento herido fácilmente cuando critican mi conducta o trabajo". Respecto a la dimensión competencias sociales las puntuaciones son altas, siendo ligeramente superiores las de los estudiantes del Grado en Educación Primaria. En dimensión regulación emocional la valoración general es media-alta, el ítem a destacar es el relativo a "Conozco mis emociones", siendo mayor la valoración en el Grado en Educación Primaria que en el Grado en Educación Infantil. Igualmente, en la dimensión conciencia emocional las valoraciones son medias, los estudiantes del Grado en Educación Primaria muestran puntuaciones ligeramente superiores a los estudiantes del Grado en Educación Infantil, excepto en el ítem "por la noche empiezo a pensar y me cuesta mucho dormirme".

Tabla 2: Resultados en la competencia emocional del alumnado en cada etapa educativa

\begin{tabular}{|l|c|c|}
\hline Dimensiones e ítems & $\begin{array}{c}\text { Educación } \\
\text { Infantil }\end{array}$ & $\begin{array}{c}\text { Educación } \\
\text { Primaria }\end{array}$ \\
\hline Dim. 1: Competencia de vida y bienestar & & \\
\hline Me siento una persona feliz & $3.92 \pm 0.80$ & $4.02 \pm 0.84$ \\
\hline${ }^{*}$ Estoy descontento conmigo mismo & $3.79 \pm 1.03$ & $3.80 \pm 1.19$ \\
\hline A menudo pienso cosas agradables sobre mí mismo & $3.38 \pm 0.99$ & $3.61 \pm 1.05$ \\
\hline Dim. $:$ : Autonomía emocional & & \\
\hline Me cuesta defender opiniones diferentes a la de las otras personas & $2.30 \pm 1.02$ & $2.26 \pm 1.09$ \\
\hline Me siento herido fácilmente cuando critican mi conducta o trabajo & $2.96 \pm 1.15$ & $2.73 \pm 1.22$ \\
\hline A menudo cuando alguien me habla estoy pensando en lo que diré yo enseguida & $2.51 \pm 1.10$ & $2.49 \pm 1.09$ \\
\hline Dim. 3: Competencias sociales & & \\
\hline Me resulta fácil darme cuenta de cómo se sienten los otros & $3.91 \pm 0.96$ & $4.03 \pm 0.90$ \\
\hline Sé ponerme en el lugar de los otros para comprenderlos bien & $4.31 \pm 0.72$ & $4.36 \pm 0.64$ \\
\hline Noto si los otros están de mal o buen humor & $4.22 \pm 0.77$ & $4.36 \pm 0.63$ \\
\hline Dim. 4: Regulación emocional & & \\
\hline Conozco bien mis emociones & $3.56 \pm 0.93$ & $4.01 \pm 0.82$ \\
\hline Acostumbro a moderar mi reacción cuando tengo una emoción fuerte & $3.13 \pm 0.90$ & $3.20 \pm 0.93$ \\
\hline${ }^{*}$ Me pongo nervioso/a con mucha facilidad y me altero & $3.15 \pm 1.16$ & $3.21 \pm 1.26$ \\
\hline Dim. 5: Conciencia emocional & & \\
\hline Puedo describir fácilmente mis sentimientos & $3.10 \pm 1.11$ & $3.29 \pm 1.07$ \\
\hline${ }^{*}$ Por la noche empiezo a pensar y me cuesta mucho dormirme & $3.17 \pm 1.33$ & $2.93 \pm 1.43$ \\
\hline Sé poner nombre a las emociones que experimento & $3.06 \pm 1.11$ & $3.29 \pm 1.08$ \\
\hline
\end{tabular}

En la figura 1, se observa el nivel de competencia emocional medio para cada una de las dimensiones del instrumento, así como en el nivel de competencia global, para cada una de las muestras (estudiantes del Grado en Educación Infantil y estudiantes del Grado en Educación Primaria). Se observa como el nivel de competencia emocional es muy similar en ambas muestras para cada dimensión, siendo ligeramente superior el desarrollo emocional del alumnado de Educación Primaria en la dimensión no 4 (regulación emocional). Por otro lado, se observa como en ambos grados, el nivel de competencia emocional en la dimensión no 2 (autonomía emocional) se encuentra entre bajo y medio. 


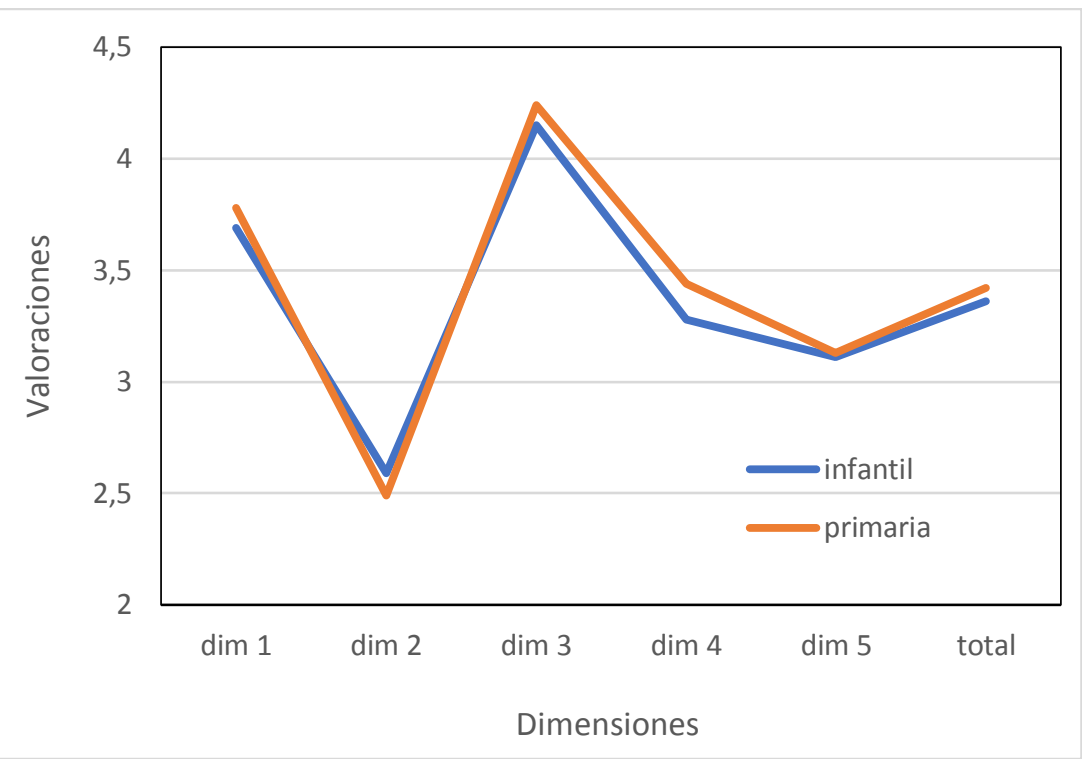

Fig. 1: Media en la competencia emocional en cada dimensión del instrumento

Para llevar a cabo el contraste estadístico en las competencias emocionales del alumnado entre ambas muestras, se comprobó la normalidad de los datos a través del test de Kolmogorov-Smirnov. Los resultados evidenciaron el supuesto de normalidad para ambas: educación infantil $(K S=0.055 ; g l=227 ; s i g .=0.097>$ $0.05)$, educación primaria $(K S=0.062 ; g l=128 ; \mathrm{sig} .=0.200>0.05)$. Ante tales resultados, se emplearon pruebas paramétricas para la comparación entre ambas distribuciones, en concreto el test t-Student para muestras independientes. Los resultados encontrados en la tabla 3 aceptaron la hipótesis nula para cada dimensión del instrumento, así como en el análisis global del instrumento, excepto para la dimensión no 4 (regulación emocional). Por lo tanto, solo fueron encontrado diferencias significativas en el nivel de competencia emocional entre el alumnado que estudia los Grados en Educación Infantil y Educación Primaria para esta dimensión $(L=0.016, \mathrm{sig} .>0.05 ; t=-2.153$, sig. $<0.05)$. Teniendo en cuenta que la diferencia de medias es significativa en el nivel 0.05 .

Tabla 3: Resutados estadísticos en relación a la competencia emocional del alumnado.

\begin{tabular}{|c|c|c|c|c|c|}
\hline & \multicolumn{2}{|c|}{ Prueba de Levene } & \multicolumn{2}{|c|}{$t$-Student } \\
\hline & & $F$ & Sig. & \begin{tabular}{l|l}
$t$ \\
\end{tabular} & Sig. \\
\hline \multirow[t]{2}{*}{ Competencia de vida y bienestar } & Se asumen varianzas iguales & 2.507 & 0.114 & -0.946 & 0.345 \\
\hline & No se asumen varianzas iguales & & & -0.922 & 0.358 \\
\hline \multirow[t]{2}{*}{ Autonomía emocional } & Se asumen varianzas iguales & 1.089 & 0.297 & 1.157 & 0.248 \\
\hline & No se asumen varianzas iguales & & & 1.141 & 0.255 \\
\hline \multirow[t]{2}{*}{ Competencias sociales } & Se asumen varianzas iguales & 4.185 & 0.042 & -1.337 & 0.182 \\
\hline & No se asumen varianzas iguales & & & -1.389 & 0.166 \\
\hline \multirow[t]{2}{*}{ Regulación emocional } & Se asumen varianzas iguales & 0.016 & 0.900 & -2.153 & $0.032^{*}$ \\
\hline & No se asumen varianzas iguales & & & -2.119 & $0.035^{*}$ \\
\hline \multirow[t]{2}{*}{ Conciencia emocional } & Se asumen varianzas iguales & 0.493 & 0.483 & -0.228 & 0.819 \\
\hline & No se asumen varianzas iguales & & & -0.226 & 0.821 \\
\hline \multirow[t]{2}{*}{ Competencia total } & Se asumen varianzas iguales & 2.042 & 0.154 & -1.201 & 0.230 \\
\hline & No se asumen varianzas iguales & & & -1.241 & 0.216 \\
\hline
\end{tabular}

\section{Resultados correlacionales entre diferentes variables}

En este apartado se analiza el efecto entre las variables seleccionadas y el nivel de competencia emocional mediante dos tipos de correlaciones. Para las variables edad, calificación de acceso al grado, calificación del 1ㅇ cuatrimestre del grado fue empleada la correlación de Pearson al ser variables cuantitativas continuas; mientras que para las variables esfuerzo hacia el estudio y motivación fue utilizada la correlación de Spearman al ser variables ordinales. En las tablas 4 y 5 se muestran los coeficientes de correlación obtenidos, y el nivel de significancia entre el nivel global de competencia emocional y las variables académicas y demográficas seleccionadas para cada muestra, en función del género del alumnado. Para el alumnado que estudia el Grado en educación Infantil, tanto para el sexo masculino como para el sexo femenino las únicas correlaciones significativas han sido las calificaciones obtenidas en el $1^{\circ}$ cuatrimestre del curso $(r=0.381 ; r=$ 
0.217) y el nivel de motivación hacia el estudio ( $r=0.307 ; r=0.237)$. Ambas correlaciones han sido positivas con un tamaño del efecto entre pequeño y moderado (tabla 4), teniendo en consideración que los resultados que presentan dos asteriscos $\left(^{* *}\right)$ poseen una correlación significativa al nivel 0.01 , mientras que los que poseen un asterisco $\left({ }^{*}\right)$ tienen un nivel de significatividad al 0.05 .

Tabla 4: Coeficientes en las correlaciones del grado de Educación infantil.

\begin{tabular}{|c|c|c|c|c|c|}
\hline $\begin{array}{c}\text { Etapa Educación } \\
\text { Infantil }\end{array}$ & Edad & $\begin{array}{c}\text { Calificación } \\
\text { Acceso }\end{array}$ & $\begin{array}{c}\text { Calificación } \\
1^{\circ} \text { Cuatrimestre }\end{array}$ & $\begin{array}{c}\text { Esfuerzo hacia el } \\
\text { estudio }\end{array}$ & $\begin{array}{c}\text { Motivación hacia el } \\
\text { estudio }\end{array}$ \\
\cline { 2 - 6 } & $r$ & $r$ & $r$ & $r$ & $R$ \\
\hline Masculino & 0.122 & 0.233 & $0.381^{* *}$ & 0.187 & $0.307^{*}$ \\
\hline Femenino & 0.085 & 0.067 & $0.217^{\star *}$ & 0.084 & $0.237^{* *}$ \\
\hline
\end{tabular}

Respecto al alumnado que estudia el Grado de Educación Primaria, se observa que para el alumnado de sexo masculino existen correlaciones significativas entre el nivel global de competencia emocional y las variables calificaciones obtenidas en el $1^{\circ}$ cuatrimestre del curso $(r=0.310)$, esfuerzo hacia el estudio $(r=$ $0.432)$ y motivación hacia el estudio $(r=0.328)$. Estas variables han sido positivas con una intensidad entre baja y moderada. Para el alumnado de sexo femenino sólo han sido dos variables las que han correlacionados significativamente, siendo éstas, el esfuerzo hacia el estudio $(r=0.403)$ y la motivación hacia el mismo $(r=$ 0.296). Ambos coeficientes también han sido positivos (Tabla 5), teniendo en cuenta que los resultados que presentan dos asteriscos $\left.{ }^{* *}\right)$ poseen una correlación significativa al nivel 0.01 , mientras que los que poseen un asterisco $\left({ }^{*}\right)$ tienen un nivel de significatividad al 0.05 .

Tabla 5: Coeficientes en las correlaciones del grado de Educación Primaria.

\begin{tabular}{|c|c|c|c|c|c|}
\hline $\begin{array}{c}\text { Etapa Educación } \\
\text { Primaria }\end{array}$ & Edad & $\begin{array}{c}\text { Calificación } \\
\text { Acceso }\end{array}$ & $\begin{array}{c}\text { Calificación } \\
1^{\circ} \text { Cuatrimestre }\end{array}$ & $\begin{array}{c}\text { Esfuerzo hacia } \\
\text { el estudio }\end{array}$ & $\begin{array}{c}\text { Motivación hacia el } \\
\text { estudio }\end{array}$ \\
\cline { 2 - 6 } & $r$ & $r$ & $r$ & $r$ & $r$ \\
\hline Masculino & 0.149 & 0.131 & $0.310^{*}$ & $0.432^{\star *}$ & $0.328^{*}$ \\
\hline Femenino & 0.132 & 0.008 & 0.071 & $0.403^{* *}$ & $0.296^{*}$ \\
\hline
\end{tabular}

\section{DISCUSIÓN}

Una vez analizados los datos se aprecia que tanto el alumnado de los Grados en Educación Infantil como en Educación Primaria poseen un nivel medio de competencia emocional, por tanto, es necesario realizar estudios de estas características para aportar orientaciones sobre las dimensiones que configuran el desarrollo emocional y el nivel de desarrollo que poseen dichos profesionales y poder plantear estrategias de mejora. Estos resultados coinciden en la muestra de estudiantes de Educación Infantil con los obtenidos por Anastasiou (2020) y con el estudio de Del Rosal et al. (2016) puesto que según este estudio, centrado en los estudiantes en Educación Primaria, dicho alumnado posee niveles superiores de desarrollo emocional a otros estudiantes universitarios. Todo ello discrepa de los resultados obtenidos por Catacora (2020) puesto que, según este autor, tanto los/as estudiantes de un grado como los del otro poseían un nivel competencial bajo y con tendencia a la baja. Pero a pesar de que los resultados obtenidos muestran un nivel competencial medio o adecuado, es pertinente seguir apostando por fortalecer el desarrollo de la competencia emocional en los grados relacionados con la Educación (Palomera et al., 2019; Pérez-Escoda et al., 2019; Schonert-Reichl, 2019) para que los/as futuros/as docentes puedan tener estilos docentes asertivos y emocionalmente competentes de acuerdo con los estudios de Ávila (2019) y Moè \& Idit (2020).

Una vez analizados los datos, se aprecia que no existen diferencias significativas entre el nivel global de la competencia emocional entre ambas muestras de estudiantes, lo cual discrepa de las conclusiones de Hernáez \& Fernández (2019), estudio en el cual los futuros maestros/as de Educación Primaría poseían niveles superiores que los futuros maestros/as de Educación Infantil. De las diferentes dimensiones analizadas en el estudio, solo se han encontrado diferencias significativas entre el alumnado del Grado de Educación Infantil y Educación Primaria en la dimensión 4 relativa a la Regulación Emocional, es decir, en la capacidad de utilizar las emociones de forma adecuada, tomando conciencia de la relación entre emoción, cognición y comportamiento. Obteniendo resultados ligeramente superiores los/as estudiantes del Grado de Educación Primaria, resultados que coinciden con los obtenidos por Del Rosal et al. (2018). En este estudio, por tanto, se evidencia que el nivel de desarrollo de las dimensiones que configuran la competencia emocional es medio, por lo que desde las instituciones de educación superior se deben proponer estrategias formativas fundamentadas en el desarrollo de la autonomía emocional, conciencia emocional y regulación emocional, principalmente. 
Al analizar las correlaciones entre el nivel de desarrollo emocional global y otras posibles variables predictoras de la misma, se evidencia que la variable edad no influye en el nivel de desarrollo de la competencia emocional, resultados que coinciden con los obtenidos por Anastasiou (2020) y contrarios a los obtenidos por Andrade et al. (2016), Dumitriu et al. (2014), y Gutiérrez (2020) puesto que según estos estudios la edad si es una variable predictora del nivel de desarrollo emocional.

En el presente estudio también se aprecia que, en el Grado en Educación Infantil, para ambos géneros, las principales variables predictoras han sido las calificaciones del primer cuatrimestre y la motivación hacia el estudio, estableciéndose correlaciones positivas, aunque con un efecto pequeño y moderado. Estos resultados corroboran los obtenidos por Del Rosal et al. (2018) y Trigueros et al. (2019) puesto que según estos estudios existen relaciones positivas entre el nivel de inteligencia emocional y el rendimiento académico. En cuanto a los/as estudiantes del Grado en Educación Primaria, en el género masculino las variables predictoras significativas fueron tres, las calificaciones del primer cuatrimestre, el esfuerzo y la motivación hacia el estudio, mientras que en el género femenino solo fueron dos variables, el esfuerzo hacia el estudio y la motivación. Teniendo en cuenta la influencia que ejercen las calificaciones del alumnado en el nivel de desarrollo emocional, es de resaltar que las calificaciones de acceso no ejercen influencia, mientras que las calificaciones del primer cuatrimestre si, lo cual puede ser debido a que el instrumento fue aplicado a principios del segundo cuatrimestre, y dichas calificaciones eran recientes.

Estos resultados coinciden parcialmente con los obtenidos por Chinuyere \& Afeez (2019) y Peregalajar \& López (2015), puesto que según estas investigaciones existen relaciones positivas entre el nivel de inteligencia emocional y el rendimiento académico. En el presente trabajo, se dice que corroboran parcialmente puesto que no se han producido correlaciones significativas en el alumnado masculino, únicamente en el femenino, coincidiendo estos resultados con los obtenidos por Deighton et al. (2019), según los cuales no hay efecto de la inteligencia emocional en el rendimiento académico.

\section{CONCLUSIONES}

Las principales conclusiones de este estudio son: 1) es necesario diseñar programas formativos ajustados a las necesidades de estos estudiantes, y que favorezcan su desarrollo socioemocional y profesional, requiriendo desarrollar una conciencia institucional sobre la importancia del desarrollo emocional de los futuros docentes de la Educación, y que consecuentemente, empiece a formar parte de los planes de estudios oficiales, dejando de estar al margen del currículum oficial; y 2) centrar el interés en el desarrollo de la competencia emocional de los/as estudiantes puede repercutir directamente en la calidad docente y en su calidad de vida, así como en la calidad del aprendizaje de los niños y niñas con los que trabajarán.

Las principales limitaciones del estudio son, por un lado, el tamaño de la muestra, puesto que sería necesario una muestra más amplia para una mayor generalización de los resultados, así como el análisis en otros grados educativos; y, por otro lado, poder realizar un estudio longitudinal donde se compruebe la influencia del currículum oculto en el desarrollo de la competencia emocional de los futuros docentes de Educación Infantil y Primaria.

\section{REFERENCIAS}

Anastasiou, S., The moderating effect of age on preschool teachers'trait emotional inteligence in Greece and implications for preschools human resources management, http://doi.org/10.18488/journal.61.2020.81.26.36, International Journal of Education and Practice, 8(1), 26-36 (2020)

Andrade, A., Martins, R., y otros tres autores, Professional factors and emotional competence in healthcare professionals, http://dx.doi.org/10.15405/epsbs.2016.07.02.40, European Proceedings of Social and Behavioural Sciences, 424-431, (2016)

Aspelin, J., Enhancing pre-service teachers' socio-emotional competence, International Journal of Emotional Education, 11(1), 153-168 (2019)

Ávila, A. M., Perfil docente, bienestar y competencias emocionales para la mejora, calidad e innovación de la escuela, https://doi.org/10.36260/rbr.v8i5.741, Revista Boletín Redipe, 8(5), 131-144 (2019)

Barraza, R., y González, M., Rendimiento académico y autopercepción de inteligencias múltiples e inteligencia emocional en universitarios de primera generación, http://dx.doi.org/10.15517/aie.v16i2.23930, Revista Actualidades Investigativas en Educación, 16(2), 1-23 (2016)

Bentler, P., EQS structural equations program manual, Multivariate software, Encino, California (1989)

Bimayu, W., Kristiawan, M. \& Fitriani, Y., The Effect of Emotional Intelligence, Student's Motivation toward Student's Achievement, International Journal of Progressive Sciences and Technologies (IJPSAT), 20(1), 6-16 (2020)

Bisquerra, R., y Pérez-Escoda, N., Las competencias emocionales, http://doi.org/10.5944/educxx1.1.10.297, Educación $\mathrm{XX1}, 10,61-82(2007)$ 
Bisquerra, R., Pérez-González, J.C., y García Navarro, E., Inteligencia emocional en educación, Síntesis, Madrid (2015)

Deighton, K., Hudson, J., y otros seis autores, Effects of emotional intelligence and supportive text messages on academic outcomes in first-year undergraduates, http://doi.org/10.1080/0309877X.2017.1377161, Journal of Further and Higher Education, 43(4), 494-507 (2019)

Denham, S.A., y Bassett, H.H., Early childhood teachers' socialization of children's emotional competence, https://doi.org/10.1108/JRIT-01-2019-0007, Journal of Research in Innovative Teaching \& Learning, 12(2), 133-150 (2019)

Dumitriu C., Timofti, I.C., y Dumitriu, G., Evaluation and development of students' emotional competence, http://doi.org/10.1016/j.sbspro.2014.01.312, Procedia - Social and Behavioral Sciences, 116, 869-874 (2014)

Escoda, N.P., Bisquerra, R., Filella, R., y Soldevilla, A., Construcción del cuestionario de desarrollo emocional de adultos (QDE-A) / Construction of the emotional development questionnaire for adults (QDE-A), Revista Española de Orientación y Psicopedagogía, 21(2), 367-379 (2010)

Estrada, M., Monferrer, D., y Moliner, M.A., El aprendizaje cooperativo y las habilidades socio-emocionales: una experiencias docente en la asignatura técnicas de ventas, http://dx.doi.org/10.4067/S0718-50062016000600005, Formación Universitaria, 9(6), 43-62 (2016)

Catacora Guzmán, V. H., Competencias emocionales y clima de clase de estudiantes de educación superior, Tesis doctoral, Universidad Cesar Vallejo, Repositorio Digital Internacional (2020)

Chinyere, O.T., y Afeez, Y.S., Influence of emotional intelligence ability level of electrical/electronic technology university students on academic motivation and attitude to study, http://doi.org/10.1177/0020720919840984, International Journal of Electrical Engineering \& Education, 24 , 1-41 (2019)

Del Rosal Sánchez, E., Dávila Acedo, M.A., Sánchez, S., y Bermejo, M.L., La inteligencia emocional en estudiantes universitarios: diferencias entre el grado de maestro en educación primaria y los grados en ciencias, http://doi.org/10.17060/ijodaep, International Journal of Developmental and Educational Psicology, INFAD Revista de Psicología, 1(1), 51-62 (2016)

Del Rosal, I., Moreno-Manso, J.M., y Bermejo, L.M., Inteligencia emocional y rendimiento académico en futuros maestros de la Universidad de Extremadura. Profesorado, Revista de Curriculum y Formación del Profesorado, 22(1), 257-275 (2018)

Gutiérrez, N., Inteligencia emocional percibida en estudiantes de educación superior: análisis de las diferencias en las distintas dimensiones, http://doi.org/10.15517/ap.v34i128.34469, Actualidades en Psicología, 34(128), 17-33 (2020)

Humphries, M.L., Williams, B.V., y May, T., Early childhood teachers' perspectives on social-emotional competence and learning in urban classrooms, http://doi.org/10.1080/15377903.2018.1425790, Journal of Applied School Psychology, 34(2), 1-23 (2018)

Hernáez, L. L., y Fernández, C.S., Formación del profesorado de magisterio, competencias emocionales según el género y etapa educativa, https://doi.org/10.4995/redu.2019.799, REDU: Revista de Docencia Universitaria, 17(1), 3 (2019)

Maguire, R., Egan, A., Hyland, P., y Maguire, P., Engaging students emotionally: the role of emotional intelligence in predicting cognitive and affective engagement in higher education, https://doi.org/10.1080/07294360.2016.1185396, Higher Education Research \& Development, 36(2), 343-357 (2017)

Moè A., y Idit K., Emotion regulation and need satisfaction shape a motivating teaching style, http://doi.org/10.1080/13540602.2020.1777960, Teachers and Teaching, 1-18 (2020)

Palomares-Ruiz, A., y Serrano-Marugán, I., Influencia de las competencias emocionales y financieras en la formación universitaria, https://dx.doi.org/10.4067/S0718-50062016000500004, Formación Universitaria, 9(5), 25-36 (2016)

Palomera, R., Briones, E., y Gómez-Linares, A., Formación en valores y competencias socio-emocionales para docentes tras una década de innovación, https://doi.org/10.19053/22160159.v10.n25.2019.9116, Praxis y Saber, 10(24), 93-117 (2019)

Pegalajar, M.C., y López, L., Competencias emocionales en el proceso de formación del docente de educación Infantil, REICE, https://doi.org/10.15366/reice, Revista Iberoamericana sobre Calidad, Eficacia y Cambio en Educación, 12 (3), 95-106 (2015)

Pérez-Escoda, N., Berlanga, V., y Alegre, A., Desarrollo de competencias socioemocionales en educación superior: evaluación del posgrado en educación emocional, https://doi.org/10.13042/Bordon.2019.64128, Bordón, Revista de Pedagogía,71(1), 97-113 (2019)

Ordun, G., y Akün, F.A., Self actualization, self efficacy and emotional intelligence of undergraduate students, http://doi.org/10.18178/joams.5.3.170-175, Journal of Advanced Management Science, 5(3), 170-175 (2017)

Rosa, G., Riberas, G., Navarro-Segura, L., y Vilar, J., El coaching como herramienta de trabajo de la competencia emocional en la formación de estudiantes de educación social y trabajo social de la Universidad Ramón Llull, España, https://dx.doi.org/10.4067/S0718-50062015000500009, Formación Universitaria, 8(5), 77-90 (2015)

Rueda, P.M., y Filella Guiu, G., Importancia de la educación emocional en la formación inicial del profesorado, https://doi.org/10.21001/ie.2016.1.17, Intercambio/Échange, 1, 212-219 (2016) 
Schonert-Reichl, K.A., Advancements in the landscape of social and emotional learning and emerging topics on the horizon, https://doi.org/10.1080/00461520.2019.1633925, Educational psychologist, 54(3), 222-232 (2019)

Trigueros, R., Aguilar-Parra, J.M., y otros cuatro autores, Influence of emotional intelligence, motivation and resilience on academic performance and the adoption of healthy lifestyle habits among adolescents,

http://doi.org/10.3390/ijerph16162810, International Journal of Environmental Research and Public Health,16, 1-12 (2019)

Zhoc, K.C., King, R.B., Chung, T., y Chen, J., Emotionally intelligent students are more engaged and successful: examining the role of emotional intelligence in higher education, https://doi.org/10.1007/s10212-019-00458-0, European Journal of Psychology of Education, 1-25 (2020) 